\title{
ALMACENAMIENTO DE CARBONO Y AGUA EN Tillandsia latifolia Meyen EN UN SECTOR DEL TILLANDSIAL DE PIEDRA CAMPANA (LIMA / PERÚ)
}

\section{CARBON AND WATER STORAGE IN Tillandsia latifolia Meyen IN A SECTOR OF THE TILLANDSIAL LOMAS PIEDRA CAMPANA (LIMA / PERU)}

\author{
Jefree Arévalo ${ }^{1}$ y Héctor Aponte ${ }^{2,3}$
}

\begin{abstract}
Resumen
Los tillandsiales son ecosistemas desérticos que se caracterizan por que sus comunidades están conformadas, principalmente, por plantas del género Tillandsia. A la fecha, se desconoce el rol que tienen estas comunidades en la captación de carbono y el potencial rol que tienen al almacenar agua en la biomasa vegetal del desierto. El presente estudio buscó cuantificar la cantidad de carbono y agua que almacena Tillandsia latifolia Meyen en un tillandsial de la costa de Lima (Piedra Campana, Cañete- Lima). Para ello, se evaluaron todas las laderas y quebradas de un sector del tillandsial, se pesó la biomasa aérea y se recolectaron muestras de suelo (primeros $10 \mathrm{~cm}$ ); se midió el porcentaje de agua y carbono almacenado en la biomasa, y el carbono en las muestras de suelo. Todos los datos obtenidos fueron procesados utilizando la técnica de interpolación kriging. El carbono total ascendió a 94.1 toneladas (t) (3.63 t/ha), hallándose más carbono almacenado en la parte del suelo (76.50 t) que en la parte aérea $(17.60$ t). La cantidad de carbono que almacena este tillandsial está por debajo del de varios ecosistemas que se utilizan para la compensación de emisiones de $\mathrm{CO}_{2}$, como humedales o formaciones vegetales amazónicas. Por otro lado, la cantidad de agua almacenada ascendió a más de 164 mil litros, valor que resulta importante como reserva de agua en esta región desértica. Se discuten las implicancias de los resultados obtenidos para la valoración y gestión de este ecosistema desértico.
\end{abstract}

Palabras clave: desierto costero, Tillandsia latifolia, tillandsial, servicio ecosistémico.

\begin{abstract}
Tillandsial lomas are desert ecosystems characterized by communities composed mainly of plants of the genus Tillandsia. The role of these communities in carbon sequestration and the potential role they have in storing water in the desert plant biomass is unknown. The present study sought to quantify the amount of carbon and water stored by Tillandsia latifolia Meyen in a tillandsial lomas of Lima (Piedra Campana, Cañete-Lima). To do this, all the slopes and ravines of a tillandsial sector were evaluated; aerial biomass was weighed and soil samples were collected (first $10 \mathrm{~cm}$ ); the percentage of water and carbon stored in the biomass, and the carbon in the soil samples were measured. All the data obtained were processed using the kriging interpolation technique. Total carbon measured was 94.10 tons (t) (3.63 t/ha) with more carbon stored in the part of the soil (76.50 t) than in the aerial part $(17.60 \mathrm{t})$. The amount of carbon stored by this tillandsial lomas is below that of several ecosystems that are used as compensation of $\mathrm{CO}_{2}$ emissions such as wetlands or Amazonian vegetation. On the other hand, the water stored amounted to 164 thousand liters, a value that is important as a water reserve in this desert region. The implications of the results obtained for the assessment and management of this desert ecosystem are discussed.
\end{abstract}

Key words: coastal desert, ecosystem service, Tillandsia latifolia, tillandsial lomas.

\section{Introducción}

Los tillandsiales son ecosistemas ubicados en zonas desérticas que reciben este nombre debido a que están dominados por especies del género Tillandsia (Pinto, 2005). Estos ecosistemas son perennes y dependen de la presencia de neblina y viento en el ambiente; ambos facilitan los nutrientes para las plantas (Pinto et al., 2006).

Las poblaciones del género Tillandsia poseen un patrón de crecimiento en forma de bandas o agrupado, patrón que depende de la cercanía de la población a la costa, de la pendiente, de la cantidad de neblina a la que están expuestas, de la topografía del lugar (ya sea en zonas arenosas o rocosas, presente en colinas o a faldas de cerros) y del transporte aéreo de los sedimentos (Hesse, 2012). El crecimiento del género Tillandsia no se da de manera uniforme en toda el área, algunas zonas tienen mayor crecimiento y distribución que otras, tal como en el caso de $T$. recurvata, que posee variaciones en el crecimiento debido a la variabilidad de las 
diferentes condiciones abióticas en las que se encuentra el área del tillandsial (por ejemplo, las diferencias en pendiente, sustrato, altura o intensidad y dirección de viento (Valverde \& Bernal, 2010). Otro caso es el de Tillandsia latifolia Meyen, cuya densidad y distribución espacial tiene relación con la topografía y el sustrato en el que se encuentran, siendo la densidad de estas plantas mayor cuando se encuentran con exposición de neblina (Aponte \& Flores, 2013).

Existen diversas amenazas para la conservación de los tillandsiales, entre ellas, la poca gestión ambiental, la creciente ampliación urbana y el cambio climático (Rundel et al., 1997; Palomino \& Cabrera, 2007; MINAM, 2014). Esto no sólo pone en riesgo el hábitat de las especies, sino también los servicios ambientales que brindan los tillandsiales. La captura de carbono es uno de los servicios importante que brindan los ecosistemas vegetales (Roulet, 2000); estimar este servicio en los tillandsiales nos permitiría dar a conocer en qué nivel contribuyen a reducir los impactos que genera el cambio climático. Otro servicio que brindan las comunidades de plantas es la provisión de hábitat a otras especies, ya que proveen refugio y/o alimento. En un ecosistema desértico, el alimento y el agua son obtenidos, también, a través del consumo de vegetales, por lo que las plantas del desierto son clave para la provisión de agua y la mantención de las especies que allí habitan (un ejemplo interesante se muestra en el trabajo de Pulido et al. (2007), donde los agroecosistemas del desierto cumplen esta función). En este contexto, estimar el agua almacenada por parte de las plantas perennes en el desierto (como las especies de Tillandsia) es importante para conocer su rol como proveedores de este recurso para los herbívoros, así como el potencial que tienen estas especies para retener el agua en el desierto.

El presente trabajo buscó estimar el carbono y el agua almacenados por Tillandsia latifolia Meyen en un sector del Tillandsial Piedra Campana (Lima, Perú), un tillandsial conocido de la ciudad de Lima, utilizando un muestreo exhaustivo y el modelamiento espacial del almacenamiento de estas sustancias en el área estudiada.

\section{Materiales y métodos}

\section{Área de estudio}

El estudio se desarrolló en el tillandsial Piedra Campana, ubicado a $1 \mathrm{~km}$ al Nor Oeste de la localidad de San Antonio, distrito de San Antonio, provincia de Cañete, Lima (UTM 18L 319503.00 E 8602952.00; Figura 1).

La región presenta un clima seco y árido, con precipitaciones entre $0-1.4 \mathrm{~mm}$ por año; temperatura media de $18.9{ }^{\circ} \mathrm{C}$, que lo caracteriza como cálido; la humedad relativa anual promedio alcanza $79 \%$ con ligeras variaciones durante el año, siendo la evaporación total media anual $942.8 \mathrm{~mm}$. Todas estas características que lo tipifican, ecológicamente, como
Desierto Sub-Tropical, extendiéndose esta formación hasta los 800 msnm (INRENA, 1995).

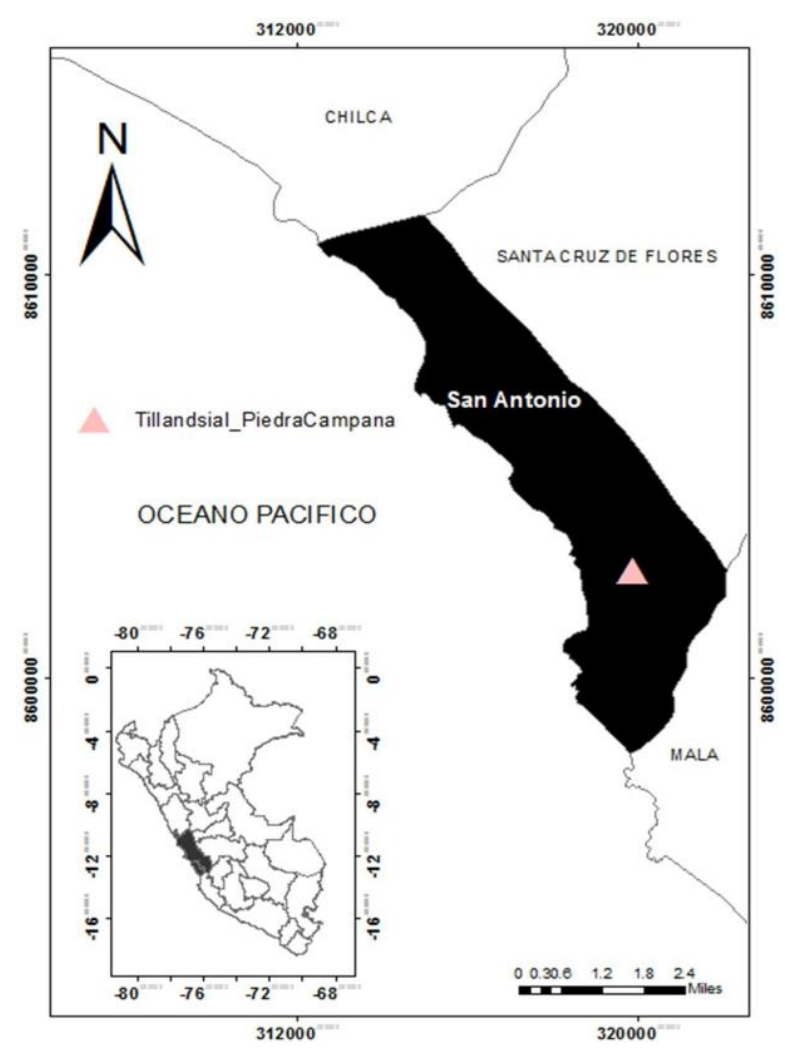

Figura 1. Mapa del área de estudio. En la esquina inferior izquierda el mapa del Perú.

El área de estudio corresponde a un sector del tillandsial de 26 hectáreas de extensión, compuesto por quebradas rocosas y laderas arenosas. En el área de estudio se encuentran únicamente plantas de Tillandsia latifolia. Otras especies (algunas cactáceas y hierbas estacionales) son muy escasas, por lo que su abundancia no fue considerada en el presente estudio. El área de estudio se diferenció en dos zonas (Figura 2). La zona 1 (Z1) rodeada por colinas con un máximo de $100 \mathrm{~m}$ de altitud que bloquean la exposición al mar, mientras que la zona 2 (Z2), también rodeada por cerros, pero donde la exposición hacia el mar es mucho mayor. La recolección de datos en campo se realizó entre las tres primeras semanas del mes de noviembre en el año 2017.

Diseño experimental

\section{Disposición de las parcelas}

Se ubicaron 72 parcelas de $100 \mathrm{~m}^{2}(10 \mathrm{~m}$ x $10 \mathrm{~m})$ distribuidas de tal forma que abarcaron las zonas: alta, media y baja de todas las laderas y quebradas del área de estudio. Dentro de cada parcela se realizaron tres sub parcelas de $1 \mathrm{~m}^{2}(1 \mathrm{~m} \times 1 \mathrm{~m})$ ubicadas de manera aleatoria (se utilizaron coordenadas obtenidas de manera aleatoria al interior de la parcela). 


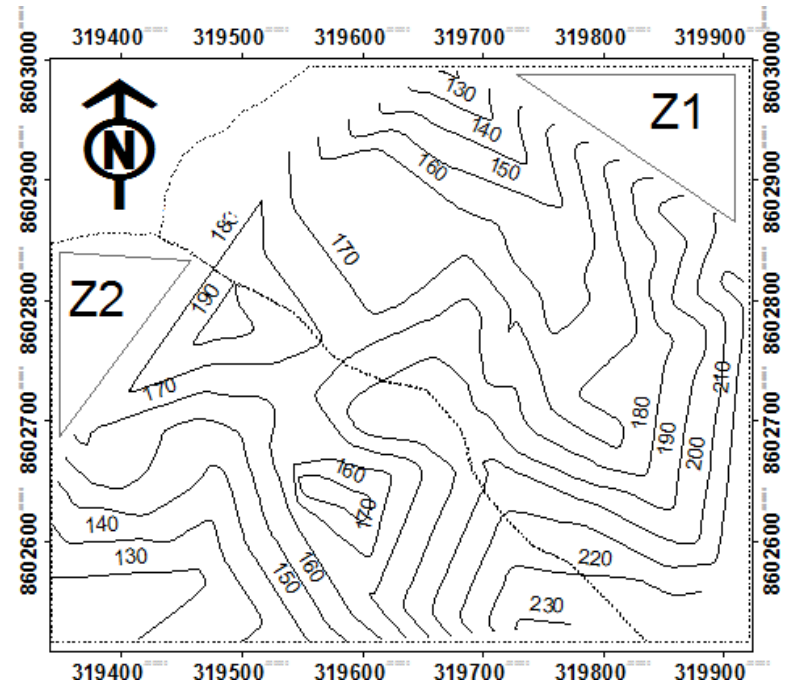

Figura 2. Detalle del área de estudio mostrando la zona 1 (Z1) y la zona 2 (Z2) divididas por una línea punteada.

Biomasa, humedad, biomasa seca y carbono en $T$. latifolia

Para conocer la biomasa, se pesaron las plantas de Tillandsia latifolia en cada subparcela. El promedio de la biomasa de las tres subparcelas permitió obtener un estimado de la biomasa por metro cuadrado en cada parcela.

Para saber el porcentaje de biomasa seca $(\% \mathrm{~S})$ en Tillandsia latifolia, se colectaron seis muestras de la especie en estudio: tres muestras de Z1 y tres de Z2, las cuales fueron procesadas secándolas en una estufa a $105{ }^{\circ} \mathrm{C}$ por un tiempo de 48 horas; luego de lo cual se midió el peso seco. La diferencia entre el peso húmedo y peso seco permitió conocer el porcentaje de humedad $(\% \mathrm{H})$. Dado que el $\% \mathrm{H}$ era el mismo en ambas zonas (se hizo una prueba preliminar sin encontrar diferencias estadísticas) y se trabajó con el promedio de las seis muestras.

El \% H fue utilizado para calcular la cantidad de agua almacenada en la biomasa. Se consideró el \%S a la diferencia obtenida de 100 - \% H. Para conocer el porcentaje de carbono en la biomasa, tres muestras (300 g cada una) de la especie se procesaron con la técnica de Walkey y Black. Esta técnica consiste en la obtención del carbono orgánico a partir de la oxidación del C producto de la reacción que se produce entre la materia orgánica (muestra) con la mezcla sulfrocrómico como lo espresa la siguiente reacción: 2 $\mathrm{Cr}_{2} \mathrm{O}_{7}=+3 \mathrm{C}^{\mathrm{o}}+16 \mathrm{H}^{+} \rightarrow 4 \mathrm{Cr}^{3+}+3 \mathrm{CO}_{2}+8 \mathrm{H}_{2} \mathrm{O}$; el C oxidado es cuantificado por medio de la titulación del exceso del $\mathrm{Cr}^{+6}$; este valor obtenido es multiplicado por el factor 1.3 para expresar los resultados en porcentaje de carbono orgánico (Walkey \& Black, 1934).

Carbono en el suelo del tillandsial

Para conocer el carbono almacenado en el suelo, se evaluaron los 10 primeros centímetros de suelo en cada parcela. La profundidad fue elegida en función al tipo de suelo en el área (el cual es principalmente rocoso, lo que en muchos casos no permite tomar una muestra a mayor profundidad que la indicada). Se obtuvieron tres muestras por parcela (200 g de cada subparcela) las cuales fueron mezcladas formando una muestra. Las muestras por parcela (72 en total) fueron evaluadas por la técnica de Walkey y Black (Walkey \& Black, 1934).

Para conocer la cantidad de suelo por metro cuadrado en los 10 primeros centímetros de profundidad, se halló la densidad aparente de las 72 parcelas, para ello se aplicó la metodología del cilindro (Blake \& Hartge, 1986). Estas muestras fueron embolsadas y llevadas al laboratorio; allí, las 72 muestras fueron pesadas para luego ser empaquetadas e introducidas en la estufa a una temperatura de $105^{\circ} \mathrm{C}$ por un tiempo de 48 horas. Posteriormente, las muestras fueron tamizadas $(2 \mathrm{~mm})$, se tomaron datos del peso seco tamizado y se obtuvo la relación de masa/volumen utilizando el volumen del barreno. La cantidad de suelo por metro cuadrado en los 10 primeros centímetros de cada subparcela (S) fue obtenida multiplicando la densidad aparente por el volumen de un prisma de $1 \mathrm{~m}$ x $1 \mathrm{~m}$ x $0.1 \mathrm{~m}$ (lado x lado x profundidad).

Análisis de datos

Análisis estadístico descriptivo

Para el análisis estadístico descriptivo se utilizó el programa Excel (ㄷ Microsoft, bajo licencia) en donde se determinaron los promedios, máximos y mínimos del contenido de carbono en cada una de las 72 parcelas y por sector del tillandsial.

\section{Cálculo del Carbono aéreo por parcela}

Para hallar la biomasa seca vegetal por metro cuadrado (B) se multiplicó la biomasa promedio de las plantas por parcela por el $\% \mathrm{~S}$. Una vez conocido B, este valor fue multiplicado por el porcentaje de carbono en la biomasa (\%CA). Para conocer la cantidad de carbono aéreo por metro cuadrado en cada parcela (CAP en $\mathrm{g} / \mathrm{m}^{2}$ ) se utilizó la Ecuación 1:

$$
\mathrm{CAP}=\mathrm{B} \times \text { \% CA ... Ecuación } 1 .
$$

\section{Cálculo del carbono en el suelo}

Conocido el porcentaje de carbono en el suelo de cada parcela (\%CS), este valor fue multiplicado por S (cantidad de suelo seco por metro cuadrado en los 10 primeros centímetros de cada parcela), obteniendo el carbono en el suelo por metro cuadrado en cada parcela $\left(\mathrm{CSP}\right.$ en $\mathrm{g} / \mathrm{m}^{2}$ ) utilizando la Ecuación 2:

$$
\mathrm{CSP}=\% \mathrm{CS} \times \mathrm{S} \ldots \text { Ecuación } 2 .
$$

\section{Modelamiento espacial}

Posteriormente, se realizaron dos modelos que permitieron conocer la cantidad de carbono del área de estudio (CT); para lo cual se utilizaron técnicas de interpolación en sistemas de información geográfica. Este último proceso se llevó a cabo utilizando un método muy similar al que usaron (Aponte \& Flores, 2013) mediante el software ARC GIS (Versión 10.3) y ArcMap 10.3. 
Para el primer modelo, se ingresó una tabla con la información de las coordenadas y con el CAP. Esta tabla fue georefenciada en la Zona UTM $18 \mathrm{~S}$. Se utilizó la herramienta kriging; el método utilizado fue el ordinario. Luego se usó la opción de modelo esférico y el modeló presentó anisotropía; la imagen proporcionada por el kriging fue exportada a raster con tamaño de celda de 1 y finalmente se cambió al formato shapefile tipo punto para que cada uno represente $1 \mathrm{~m}^{2}$ y se procedió a sumar, dando como resultado el carbono total aéreo (CA).

Para el segundo modelo se realizó el mismo procedimiento con los datos de CSP y se halló el carbono de suelo total (CS).

La cantidad de carbono en el área de estudio (CT) se obtuvo utilizando la Ecuación 3:

$$
\mathrm{CT}=\mathrm{CA}+\mathrm{CS} \ldots \text { Ecuación } 3 .
$$

Con el carbono aéreo total (CA), el porcentaje de carbono en la biomasa (\%CA), el porcentaje húmedo de la planta $(\% \mathrm{H})$ y el porcentaje seco de la planta $(\% \mathrm{~S})$, se estimó la cantidad de agua (A) en la biomasa total del tillandsial, utilizando la Ecuación 4:

$$
\mathrm{A}=(\mathrm{CA} \times \% \mathrm{H}) /(\% \mathrm{CA} \times \% \mathrm{~S}) \text {... Ecuación } 4 .
$$

\section{Resultados}

Humedad, porcentaje de carbono y biomasa

El promedio de la humedad en la biomasa aérea fue de $83.09 \%(81.8 \%$ - 84.76\%). Los valores del porcentaje de carbono en la biomasa promedio fue $52.66 \%(52.43 \%-53.04 \%)$. El promedio de la biomasa en las 72 parcelas fue de $668.52 \mathrm{~g} / \mathrm{m}^{2}\left(0-4083.3 \mathrm{~g} / \mathrm{m}^{2}\right)$. Porcentaje de carbono y análisis del suelo en las 72 parcelas

El promedio del porcentaje de carbono en el suelo por metro cuadrado fue de $0.065 \%(0.02-0.33 \%)$. El promedio en gramos de suelo por metro cuadrado de suelo de las 72 parcelas fue de $4.6 \times 105 \mathrm{~g} / \mathrm{m}^{2},(8.9 \times 105-$ $2 \times 105 \mathrm{~g} / \mathrm{m}^{2}$ ).

Cantidad de carbono aéreo y del suelo por parcela

El promedio de carbono aéreo de las 72 parcelas es de $59.53 \mathrm{~g} / \mathrm{m}^{2}\left(0-363.61 \mathrm{~g} / \mathrm{m}^{2}\right)$. El promedio de carbono en el suelo de las 72 parcelas fue de 305.21 $\mathrm{g} / \mathrm{m}^{2}\left(69.56-1524.10 \mathrm{~g} / \mathrm{m}^{2}\right)$.

Modelamiento del carbono total aéreo y del suelo en el área de estudio

La cantidad estimada de carbono aéreo es de 17.6 toneladas $(\mathrm{t})$. El promedio de todos los datos estimados por el modelo es de $68.73 \mathrm{~g} / \mathrm{m}^{2}\left(0-352.88 \mathrm{~g} / \mathrm{m}^{2}\right.$; desviación estándar (DS $\left.=61.78 \mathrm{~g} / \mathrm{m}^{2}\right)$ de carbono. También se estimó que la $\mathrm{Z} 1$ tiene $6 \mathrm{t}$ de carbono almacenado, su promedio es de $40.23 \mathrm{~g} / \mathrm{m}^{2}$ (0 - 156.68 $\left.\mathrm{g} / \mathrm{m}^{2} ; \mathrm{DS}=30.43 \mathrm{~g} / \mathrm{m}^{2}\right)$, mientras que en la $\mathrm{Z2}$ se estimaron $11.6 \mathrm{t}$ de carbono almacenado, el promedio de los datos obtenidos es de $108.99 \mathrm{~g} / \mathrm{m}^{2}$ (0 - 352.88 $\mathrm{g} / \mathrm{m}^{2} ; \mathrm{DS}=71.62 \mathrm{~g} / \mathrm{m}^{2}$ ). La Figura 3 muestra el mapa obtenido a partir de este modelamiento.

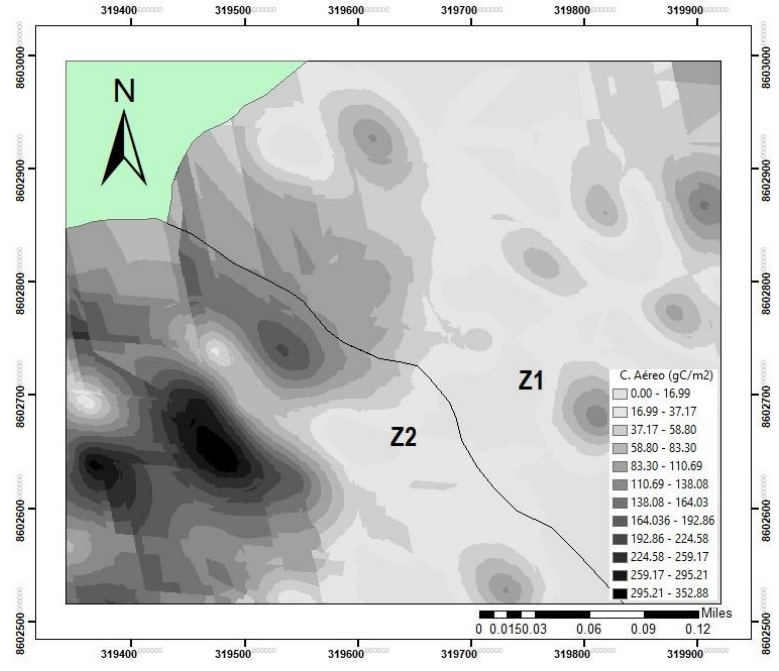

Figura 3. Mapa de carbono aéreo en el área de estudio. Los colores oscuros representan las áreas con mayor carbón almacenado en las Tillandsias.

La cantidad estimada de carbono de suelo es de 76.5 t. El promedio de todos los datos estimados es de $294.99 \mathrm{~g} / \mathrm{m}^{2}\left(94.55\right.$ - $\left.1100.53 \mathrm{~g} / \mathrm{m}^{2} ; \mathrm{DS}=187.74 \mathrm{~g} / \mathrm{m}^{2}\right)$ de carbono. También se estimó que la $\mathrm{Z} 1$ tiene $34.4 \mathrm{t}$ de carbono almacenado, su promedio es de $225.67 \mathrm{~g} / \mathrm{m}^{2}$ $\left(94.55-688.43 \mathrm{~g} / \mathrm{m}^{2}\right.$; DS $\left.=98.17 \mathrm{~g} / \mathrm{m}^{2}\right)$, mientras que en la Z2 tiene $42.1 \mathrm{t}$ de carbono almacenado, el promedio de los datos obtenidos es de $\mathrm{g} / \mathrm{m}^{2}$ (106.04 $1100.53 \mathrm{~g} / \mathrm{m}^{2}$; DS $=234.78 \mathrm{~g} / \mathrm{m}^{2}$ ). La Figura 4 muestra el mapa obtenido a partir de este modelamiento.

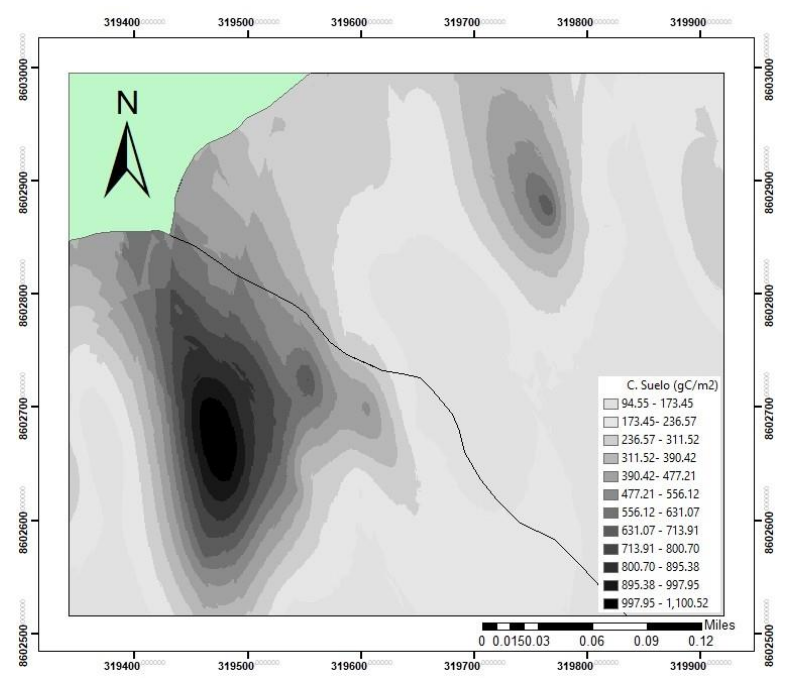

Figura 4. Mapa de carbono del suelo en el área de estudio. Los colores oscuros representan las áreas con mayor carbón almacenado en las Tillandsias.

Sumado los datos de carbono aéreo y de suelo en el área de estudio se obtiene que en este tillandsial se almacenan 94.10 toneladas de carbono. 
En el caso del agua, se estimó que la cantidad de agua del tillandsial es de $164.52 \mathrm{t}$, que equivalen a 164 5201.

\section{Discusión}

Cantidad de carbono almacenado por hectárea

Comparando con otros valores de almacenamiento de carbono conocidos para otros ecosistemas, la capacidad de almacenamiento de este tillandsial está por debajo de la capacidad de almacenamiento de comunidades como por ejemplo algunas plantas presentes en los humedales como muestra la Tabla 1.

Tabla 1. Almacenamiento de carbono en diferentes especies y formaciones vegetales.

\begin{tabular}{|c|c|c|}
\hline Especie & $\begin{array}{l}\text { Almacenamiento } \\
\text { de Carbono (t/ha) }\end{array}$ & Referencia \\
\hline $\begin{array}{l}\text { Schoenoplectus } \\
\text { californicus }\end{array}$ & 28.9 & \multirow{4}{*}{$\begin{array}{c}\text { Palomino \& } \\
\text { Cabrera, 2007 }\end{array}$} \\
\hline $\begin{array}{l}\text { Schoenoplectus } \\
\text { americanus }\end{array}$ & 18.6 & \\
\hline $\begin{array}{l}\text { Distichlis } \\
\text { spicata }\end{array}$ & 17 & \\
\hline $\begin{array}{l}\text { Sarcocornia } \\
\text { neei }\end{array}$ & 6.1 & \\
\hline $\begin{array}{l}\text { Eichhornia } \\
\text { azurea }\end{array}$ & $3.5-17.5$ & Coutinho, 1989 \\
\hline Nymphoides & 7.6 & Menezes, 1984 \\
\hline $\begin{array}{l}\text { Nymphoides } \\
\text { rudgeana }\end{array}$ & 3.8 & $\begin{array}{c}\text { Camargo \& } \\
\text { Florentino, 2000 }\end{array}$ \\
\hline $\begin{array}{l}\text { Paspalum } \\
\text { fasciculatum }\end{array}$ & 70 & $\begin{array}{c}\text { Junk \& Piedade, } \\
1993 \\
\end{array}$ \\
\hline $\begin{array}{l}\text { Paspalum } \\
\text { repens }\end{array}$ & 31 & \multirow{4}{*}{ Menezes, 1984} \\
\hline $\begin{array}{l}\text { Luziola } \\
\text { spruceana }\end{array}$ & 7.6 & \\
\hline Oryza perennis & 27 & \\
\hline $\begin{array}{l}\text { Pontederia } \\
\text { cordata }\end{array}$ & $3.8-9.7$ & \\
\hline $\begin{array}{l}\text { Formaciones } \\
\text { vegetales } \\
\text { amazónicas }\end{array}$ & 776000 & $\begin{array}{c}\text { Martel \& } \\
\text { Cairampoma, } \\
2012 \\
\end{array}$ \\
\hline $\begin{array}{l}\text { Bosques } \\
\text { templado- } \\
\text { lluviosos } \\
\text { (región centro- } \\
\text { sur de Chile) } \\
\text { pre cordillera de } \\
\text { los Andes }\end{array}$ & 662.06 & \multirow{2}{*}{ Schlegel, 2001} \\
\hline $\begin{array}{l}\text { Bosques } \\
\text { templado- } \\
\text { lluviosos } \\
\text { (región centro- } \\
\text { sur de Chile) } \\
\text { Cordillera de la } \\
\text { Costa }\end{array}$ & 423.86 & \\
\hline $\begin{array}{l}\text { Tillandsia } \\
\text { latifolia Meyen }\end{array}$ & 3.6 & presente trabajo \\
\hline
\end{tabular}

Esto significa que el servicio ecosistémico de almacenamiento de carbono en este tillandsial es menor que el que brindan las especies y ecosistemas citados en la bibliografía precedente. Esto no significa que el tillandsial no brinda el servicio de almacenamiento de carbono, sino que este servicio es menor. Una de las principales razones por las que podría explicarse esta situación es porque la estrategia de vida de la especie en estudio consiste en acumular agua (más del $80 \%$ de la planta es agua), lo que representa un valor bastante bajo de biomasa seca. A ello podemos agregar que los estudios citados en la Tabla 1, tienen como características una alta productividad de biomasa, mejor almacén de la descomposición de la materia orgánica, mayor disponibilidad de agua y presencia de precipitaciones, lo que contribuye a que tengan un mayor potencial como sumideros de carbono, en comparación al área de estudio del presente trabajo; ya que esta última presenta un mayor estrés hídrico y tiene completa dependencia de la neblina, lo que tiene como resultado un crecimiento lento (Pinto, 2005) y en consecuencia una menor producción de biomasa (lo que significa menos conversión del carbono atmosférico a carbono orgánico). Los resultados obtenidos nos indican que este ecosistema no es óptimo para su consideración dentro del mercado de bonos de carbono (ya que presenta muy poco carbono almacenado por hectárea). Debemos tener en cuenta que el presente estudio consideró solo un sector del tillandsial, lo que significa que todo el tillandsial completo puede brindarnos un mayor almacenamiento de carbono, aunque (de tener un patrón similar al mostrado en este estudio) con baja cantidad de carbono por metro cuadrado. Para comprobar esta hipótesis debería realizarse un muestreo más detallado de las áreas adyacentes.

Distribución del carbono en el área de estudio

Los resultados muestran que existe más carbono almacenado en el suelo que en la parte aérea (76.50 y

$17.60 \mathrm{t}$, respectivamente). Es usual que el suelo sea el medio donde generalmente se almacena más carbono, y su cantidad va disminuyendo a mayor profundidad (Salvia et al., 2012). La cantidad de carbono almacenado en el suelo no es uniforme en toda el área estudiada (cada parcela tenía una cantidad distinta de carbono almacenado, Figura 4). El aporte que genera la materia vegetal muerta al suelo es la principal fuente de carbono en este estrato (Hernández et al., 2014); donde hay mayor densidad de biomasa viva hay más crecimiento y mortandad, en consecuencia, mayor cantidad de carbono almacenado en suelo. La zona donde hay mayor cantidad de carbono en el suelo también es la misma zona donde hay mayor cantidad de carbono aéreo, y es también la zona de mayor densidad poblacional; la densidad de Tillandsia latifolia en el área de estudio depende del sustrato, exposición a la neblina, la cantidad de luz al día y orientación (los cuales son diferentes en cada zona del tillandsial) y su distribución depende de la topografía y el sustrato del área de estudio, siendo más abundante en 
las laderas con cara hacia el mar (suroeste) y en sustratos arenoso de poca pendiente. (Aponte \& Flores, 2013); en consecuencia, en las zonas de mayor densidad habrá más carbono almacenado.

Almacenamiento de agua

$\mathrm{Si}$ bien es cierto que la cantidad de carbono almacenado no es mayor ni semejante a los almacenados en ecosistemas previamente comparados, la cantidad de agua almacenada asciende a varios cientos de miles de litros (164 520 mil 1, para ser exactos) lo que revela el importante rol de estos ecosistemas como fuentes de agua en el desierto. Es fundamental la presencia de agua dentro de un ecosistema, pues se sabe que es un componente químico fundamental para los organismos (Rico \& Pérez, 1997). El agua almacenada por Tillandsia latifolia sería el sustento para las aves, insectos, mamíferos y otros organismos que puedan depender de ella en esta región desértica, como podría ser la lechuza de los arenales (Athene cunicularia), la "lagartija de los arenales" (Microlophus theresiae), insectos (Lepidoptera e Hymenoptera) o himenópteros, y a los colibríes (principales visitantes y polinizadores de Tillandsia latifolia) (Aponte \& Flores, 2013), quienes consumen las hojas, semillas y el néctar de la planta.

La neblina es la principal fuente de agua de las tillandsias. Sería de gran aporte poder conocer la captación de agua de neblina por día de $T$. latifolia, tal como se tiene cuantificado para Tillandsia landbeckii (entre 2.5 y 3.7 1/m²/día) (Westbeld, et al., 2009). El presente estudio muestra que hay un potencial grande en la captura de agua de neblina. La forma en la que $T$. latifolia y otras especies del género captan el agua de neblina es a través de los tricomas (apéndices epidérmicos en forma de pelo) que se encuentran en sus hojas; estos también les permite tener una protección contra el sol y la transpiración (Alonso, 2011). Conocer los mecanismos y compuestos químicos que tienen estos tricomas podría ser de interés en la aplicación a las tecnologías que hoy en día se usan, para su mejoramiento en la captación de agua de neblina, y sea usado como fuente de agua.

La creciente y desordenada ampliación urbana amenaza la conservación de este hábitat. El conocimiento de los niveles de almacenamiento de carbono y agua en los ecosistemas desérticos, y específicamente en cada formación vegetal, permite un mejor criterio de decisión y manejo en las zonas de conservación. Los resultados del presente estudio permiten brindarnos un panorama con respecto al almacenamiento del carbono y agua para entender el rol de los tillandsiales como proveedores de servicios ecosistémicos.

\section{Agradecimientos}

A Junior, Manuel, Gabriela, Daniella y Alessandra por el apoyo en la obtención de datos en el área de estudio. A Violeta por la ayuda a nivel de laboratorio.
A Jhon Flores, por la asesoría con el software ArcGis. Finalmente, agradecemos a la Universidad Científica del Sur por el soporte logístico y el préstamo de los laboratorios de Ingeniería Ambiental y Oceanografía. El presente trabajo formó parte de la Tesis para obtención del Título de IngenieroAambiental de Jefree Arévalo.

\section{Literatura citada}

Alonso J. R. 2011. Manual de Histología Vegetal. Salamanca: Editorial Paraninfo.

Aponte H. \& Flores J. 2013. Densidad y distribución espacial de Tillandsia latifolia en el tillandsial de Piedra Campana (Lima, Perú). Ecología Aplicada, 12: 35-43.

Blake G. \& Hartge K. 1986. Bulk Density. In: Klute A. (Ed) Methods Of Soil Analysis. Part 1. Physical and Mineralogical Methods. American Society of Agronomy, Soil Science Society of America, Madison, Wisconsin, Usa. 363-375.

Camargo A. \& Florentino E. 2000. Population dynamics and net primary production of the aquatic macrophite Nymphaea rudgeana C. F. Mey in a lotic environment of the Itanhaém river basin (Sp, Brazil). Revista Brasileira de Biologia, 60: 83-92.

Coutinho M. 1989. Ecologia populacional de Eichhornia azurea e sua participacao na dinamida da vegetacao aquática da Lagoa do Infernao, SP. Dissertacao (Maestrado), Universidade Federal de Sao Carlos, Brasil.

Hernández J.E., Tirado D. \& Beltrán I. 2014. Captura de carbono en los suelos. Padi Boletín científico del ICBI, Universidad Autónoma del Estado de Hidalgo.

Hesse R. 2012. Spatial distribution of and topographic controls on tillandsia fog vegetation in coastal southern Peru: remote sensing and modelling. Journal Of Arid Environments, 78: 33-40.

INRENA (Instituto Nacional de Recursos Naturales / PE). 1995. Estudio de factibilidad para la derivación de las aguas del río mala a la cuenca del río Chilca. Lima, Perú.

Junk W. \& Piedade M. 1993. Biomass and primaryproduction of herbaceous plant communities in the amazon floodplain. Hydrobiologia, 263: 155-162.

Martel C. \& Cairampoma L. 2012. Cuantificación del carbono almacenado en formaciones vegetales amazónicas en "Cicra", Madre De Dios (Perú). Ecología Aplicada, 11: 59-65.

Menezes C. 1984. Biomassa e producao primária de tres espécies de macrófitas aquáticas da represa Do Lobo (Broa). Dissertacao (Maestrado), Universidade Federal De Sao Carlos, Brasil.

MINAM (Ministerio del Ambiente / PE). 2014. Estrategia nacional ante el cambio climático. Ministerio del Ambiente. Lima.

Palomino D. \& Cabrera C. 2007. Estimación del servicio ambiental de captura de $\mathrm{CO}_{2}$ en la flora de los humedales de Puerto Viejo. Instituto de Investigación Figmmg, 10: 49-59.

Pinto P., Barría I. \& Marquet P. 2006. Geographical distribution of tillandsia lomas in the Atacama desert, northern Chile. Journal Of Arid Environments, 65: 543552.

Pinto R. 2005. Tillandsia del norte de Chile y del extremo sur del Perú. Gas Atacama, Chile. 
Pulido V., Salinas L. \& Arana C. 2007. Aves en el Desierto de Ica. La experiencia de Agrokasa. AGROKASA, Lima, Perú.

Rico Galicia A. \& Pérez Orta R.E. 1997. Quimica I. Agua y Oxígeno. Mexico D.F.: UNAM.

Roulet N. 2000. Peatlands, Carbon Storage, Greenhouse Gases, and The Kyoto Protocol: Prospects And Significance For Canada. Wetlands, 20: 605-615.

Rundel P., Palma B., Dillon M., Rasoul M., Nilsen E. \& Boonpragob K. 1997. Tillandsia landbecki in the coastal Atacama desert of northern Chile. Revista Chilena de Historia Natural, 70: 341-349.

Salvia M., Ceballos D., Grings F., Karszenbaum H. \& Kandus P. 2012. Post-Fire effects in wetland environments: landscape assesment of plant coverage and soil recovery in the Paraná river delta marshes,Argentina.FireEcology, 8(2):17-37.DOI:

https://doi.org/10.4996/fireecology.0802017.
Schlegel B. 2001. Estimación de la biomasa y carbono del tipo forestal siempreverde. Universidad Austral De Chile.

Valverde T. \& Bernal R. 2010. ¿Hay asincronía demográfica entre poblaciones locales de Tillandsia recurvata? Evidencias de su funcionamiento metapoblacional. Boletín de la Sociedad Botánica de México, 86: 23-26.

Walkey A. \& Black I. 1934. An examination of the Degtjareff method for determining soil and, and a modification of the chromic acid titration method. Soil Science, 37: 2938.

Westbeld A., Klemm O., Grießbaum F., Sträter E., Larrain H., Osses P. \& Cereceda P. 2009. Fog deposition to a Tillandsia carpet in the Atacama desert. Annales Geophysicae, 27(9): 3571-357. DOI: https://doi.org/10.5194/angeo-27-3571-2009.

${ }^{1}$ Carrera de Ingeniería Ambiental / Facultad de Ciencias Ambientales / Universidad Científica del Sur. Av. Antigua Carretera Panamericana Sur km 19 Villa El Salvador. - Lima 42, Perú. Email: je-free@ hotmail.com.

${ }^{2}$ Carrera de Biología Marina / Facultad de Ciencias Veterinarias y Biológicas / Universidad Científica del Sur. Av. Antigua Carretera Panamericana Sur, km 19, Villa El Salvador. Lima 42 / Perú. Email: haponte@cientifica.edu.pe.

${ }^{3}$ Laboratorio de Florística / Departamento de Dicotiledóneas / Museo de Historia Natural / Universidad Nacional Mayor de San Marcos. Avenida Arenales 1256, Jesús María / Lima. Apartado 14-0434, Lima 14 / Perú. 